\title{
ATENCIÓN SOCIOSANITARIA DE LOS ADULTOS MAYORES EN EL PERÚ
}

\author{
Paola Casas-Vasquez ${ }^{1,2, a}$, Rossana Apaza-Pino ${ }^{3, b}$, Juan del Canto y Dorador ${ }^{1,4, c}$, Helver Chávez-Jimeno ${ }^{1,2, c}$ \\ RESUMEN \\ Los cambios demográficos y epidemiológicos han conllevado a la reestructuración de los sistemas de salud a nivel \\ mundial y local, cambios que incluyen un enfoque de cobertura más amplio, en el que se da importancia no solo a la parte \\ del bienestar físico de las personas, sino también a temas como el bienestar social, todo ello encaminado a asegurar un \\ envejecimiento saludable. En esta revisión se analiza el enfoque sociosanitario actual desde las diferentes instituciones a \\ cargo de población adulta mayor y los cambios que han generado en el abordaje y atención de dichas personas.
}

Palabras clave: Atención médica; Envejecimiento. (fuente: DeCS BIREME).

\section{SOCIAL HEALTH CARE FOR OLDER ADULTS IN PERU}

\begin{abstract}
Demographic and epidemiological changes have led to restructuring of the local and global health systems, changes that focus on broader coverage in which importance is given to an individual's physical well-being as well as social welfare in an effort to ensure healthy aging. In this review, the current social health care approach is analyzed from the different institutions that care for older populations and the changes that have resulted from boarding and caring for older adults.
\end{abstract}

Keywords: Medical care; Aging. (source: MESH NLM).

\section{SISTEMAS SOCIOSANITARIOS Y ASPECTOS NORMATIVOS EN EL PERÚ}

Los sistemas sanitarios han estado, tradicionalmente, enfocados en atender patologías agudas y necesidades urgentes de examinar, diagnosticar, aliviar síntomas y/o curar. Sin embargo, se requiere un abordaje diferente en la salud de la población. Un enfoque que responda a los cambios que experimentamos por causa de la transición demográfica y epidemiológica, el consecuente incremento de la esperanza de vida así como la prevalencia de enfermedades crónicas; sobre todo en la población adulta mayor, que suele tener características muy particulares ${ }^{(1)}$.

A nivel internacional, con la Organización Mundial de la Salud (OMS) como líder, los países han considerado necesario ampliar los servicios no solo centrados en lo sanitario, sino también en los servicios sociales y comunitarios, vinculando para ello a la persona y su entorno sociofamiliar (2). Esta ampliación implica la modificación de paradigmas culturales en relación a la orientación de la atención, la visión de la enfermedad -muchas veces tubular-, para centrarse en la persona y en sus diferentes esferas biopsicosociales, lo que se ejemplifica en la tabla $1^{(3)}$.

Por otro lado, se plantean elementos que definen a una atención sociosanitaria adecuada (2):

- Responde a situaciones de necesidad compleja que requieren de intervenciones de naturaleza mixta, social y sanitaria, simultánea o secuencial; en todo caso, complementarias y articuladas.

- Tiene por objetivo principal, garantizar la continuidad de cuidados, evitando desajustes materiales y temporales.

- Recurre para su prestación a estrategias e instrumentos de colaboración que se articulan interdisciplinariamente.

\footnotetext{
Instituto de Gerontología, Universidad Peruana Cayetano Heredia. Lima, Perú.

Hospital Nacional Cayetano Heredia. Lima, Perú.

Seguro Social de Salud. Lima, Perú.

Ministerio de Salud. Lima, Perú.

Médico geriatra; ${ }^{\mathrm{b}}$ médico geriatra, magíster en Salud Pública; ${ }^{\mathrm{c}}$ médico especialista en Medicina Preventiva, magíster en Salud Pública; ${ }^{\mathrm{d}}$ médico geriatra magister en salud pública

Recibido: 07/03/2016 Aprobado: 01/06/2016
}

Citar como: Casas-Vasquez P, Apaza-Pino R, del Canto y Dorador J, Chávez-Jimeno H. Atención sociosanitaria de los adultos mayores en el Perú. Rev Peru Med Exp Salud Publica. 2016;33(2)351-6. doi: 10.17843/rpmesp.2016.332.2212 
Tabla 1. Cambios culturales para la articulación del nuevo modelo de atención sociosanitario (basado en recomendaciones de OMS)

\begin{tabular}{lll}
\hline Cultura & \multicolumn{1}{c}{$\begin{array}{c}\text { Modelo sanitario } \\
\text { (enfoque actual) }\end{array}$} & \multicolumn{1}{c}{$\begin{array}{c}\text { Modelo sociosanitario } \\
\text { (nuevo enfoque) }\end{array}$} \\
\hline Paradigma & Curar & Curar y cuidar \\
Orientación & Centrado en la enfermedad & Centrado en la persona \\
& Diagnóstico y tratamiento & $\begin{array}{l}\text { Diagnóstico, tratamiento, cuidados y au- } \\
\text { tocuidados } \\
\text { Valoración integral (médica, funcional, } \\
\text { Social) }\end{array}$ \\
\hline Funcionalidad/discapacidad & Hiperespecialización & Atención a discapacidad y dependencia \\
\hline Organización & No considerado & Multidisciplinar/basado en procesos \\
\hline
\end{tabular}

Adaptado de Avellaneda A, Izquierdo M, Torrent-Farnel J, Ramón JR. Enfermedades raras: enfermedades crónicas que requieren un nuevo enfoque sociosanitario. An Sist Sanit Navar. 2007;30(2):177-90

- Se enfocan en la atención integral centrada en la persona orientada a garantizar el máximo nivel de bienestar, calidad de vida y autonomía.

Nuestro país sufre también los cambios demográficos y epidemiológicos antes mencionados. Las proyecciones del Instituto Nacional de Estadística e Informática (INEI) observan que para el año 2015, la población adulta mayor superó los 3 millones, y para el 2050 se proyecta en 8,7 millones, con predominio del sexo femenino (4). Esta realidad ha planteado desafíos en término de políticas y programas sociales basados en normativas jurídicas establecidas, tales como:

- Ley 28803 (5), Ley de las Personas Adultas Mayores. Es un documento marco que "garantiza los mecanismos legales para el ejercicio pleno de los derechos de las personas adultas mayores reconocidas en la Constitución Política y los tratados internacionales vigentes; orientados a mejorar su calidad de vida e integración plena al desarrollo social, económico, político y cultural".

En su estructura, enfatiza el desarrollo de programas que permitan mantener vínculo entre la salud física y mental o social. Así, en su artículo 8, promueve la creación de centros de integrales de atención al adulto mayor (CIAM) en municipalidades distritales y provinciales, con el fin de: desarrollar lazos de mutuo conocimiento y amistad entre participantes, identificar problemas familiares o individuales, combatir y prevenir los problemas de salud más comunes en este grupo de edad; realizar actividades de carácter integrativo y recreativo, participar de talleres de autoestima, mantenimiento de funciones cerebrales superiores y prevención de enfermedades crónicas, otorgar importancia a la alfabetización, implementar talleres de manufactura y desarrollo de habilidades laborales puntuales, y promover en la ciudadanía el trato diligente, respetuoso y solidario con las personas adultas mayores.

De igual manera, y no menos importante, promueve que hayan instalaciones públicas adecuadas en infraestructura para las personas adultas mayores, lo cual se señala en el artículo 12: las municipalidades dicten las disposiciones necesarias para este efecto así como el Ministerio de Transportes y Comunicaciones en relación a los vehículos de transporte público que deben disponer de equipos y accesorios adecuados en pro de la seguridad del adulto mayor.

- Política Nacional en relación a las Personas Adultas Mayores. Aprobado por el Decreto Supremo 011-2011-MIMDES, que plantea cuatro lineamientos de políticas públicas ${ }^{(6)}$ :

- Lograr el envejecimiento saludable, mejorando el bienestar físico, psíquico y social de esta población, garantizando su asistencia nutricional y alimentaria, y la generación de oportunidades para una vida armónica dentro de su familia y comunidad.

- Promover oportunidades, mediante mecanismos que permitan mejorar su calificación e inserción en el mercado laboral, garantizando un nivel mínimo de ingresos y su acceso a servicios de previsión y seguridad social.

- Incrementar sus niveles de participación social y política, de manera que sean integrados en la sociedad como agentes de desarrollo.

- Elevar el nivel educativo y cultural, promoviendo una imagen positiva del envejecimiento, e incrementar en la sociedad el reconocimiento hacia sus valores y experiencias.

Todas estas acciones y cambios; sin embargo, son de difícil aplicación, debido a la segmentación en la atención 
de salud que existe en nuestro país, pues dificultan la labor rectora del Ministerio de Salud (MINSA) y facilita la duplicidad de acciones, lo que genera muchas veces una falta de articulación en las acciones en los diferentes niveles de atención del país. Esto se refleja en el aseguramiento de salud de la población adulta mayor. De acuerdo a la información brindada por la Encuesta Nacional de Hogares (ENAHO). ${ }^{(7)}$ para el 2014 , el $75,5 \%$ de las personas adultas mayores contaban con algún tipo de seguro en salud, esto es, 2982 142; de ellas, el $53 \%$ accede al Seguro Integral de Salud (SIS) y $47 \%$ al Seguro Social de Salud del Perú (EsSalud). Para fines de este mismo año ya se contaba con 78 establecimientos de salud de nivel II y III, con servicios de geriatría, de los cuales 44 eran privados, 14 de gobiernos regionales, 11 de EsSalud, 5 del MINSA, 3 de las Fuerzas Armadas (FFAA) y policiales (PNP) y 1 en otros ${ }^{(7)}$.

Con toda esta problemática conocida previamente, ya desde el año 2012, el MINSA, EsSalud, las fuerzas armadas y policiales y el Ministerio de la Mujer y Poblaciones Vulnerables (MIMP) iniciaron el desarrollo de un conjunto de programas y acciones de promoción, prevención y atención de salud integral dirigidos a mejorar la salud física y mental de las personas adultas mayores, promoviendo el envejecimiento saludable, con lo que los servicios sociosanitarios están aumentando en cobertura y calidad.

Dentro de los programas sociales creados por el gobierno están:

- Programa Nacional de Asistencia Solidaria (Pensión 65) ${ }^{(8)}$. Programa a cargo del Ministerio de Desarrollo e Inclusión Social (MIDIS) que tiene por objetivo "dotar de un ingreso económico periódico que atenúe la vulnerabilidad social de las personas adultas mayores en condición de extrema pobreza, con la finalidad de mejorar su calidad de vida". A julio de $2014{ }^{(9)}$, se registraron 380000 usuarios en todo el territorio nacional, lográndose afiliar hasta entonces al $82,41 \%$ del total de adultos mayores en extrema pobreza que viven en los 202 distritos calificados con más del $50 \%$ de extrema pobreza.

- Programa Nacional Vida Digna (10). Programa que tiene por objetivo restituir y proteger los derechos fundamentales de las personas adultas mayores en situación de calle. Su ejecución es realizada de manera conjunta por el Ministerio de Mujer y Poblaciones Vulnerables (MIMP), los gobiernos locales y las Sociedades de Beneficencia Pública. De diciembre de 2012 a la actualidad, se han beneficiado 513 personas adultas mayores en situación de calle, de los cuales 399 han sido acogidos en el servicio de atención integral y 114 en el servicio de atención básica (hospedería).
En el 2015 se han realizado 7425 atenciones especializadas en psicología, evaluación social, de salud, y recreacionales. A la fecha se interviene en las siguientes ciudades: Arequipa, Ica, Huánuco, Huaraz, Lima y Tacna ${ }^{(11)}$.

\section{ATENCIÓN SOCIOSANITARIA}

La OMS menciona que: las actividades que las personas valoran difieren entre los individuos y cambian a lo largo de la vida ${ }^{(12)}$, y en este sentido, las personas mayores consideran importante tener una identidad, un rol, las relaciones humanas, la autonomía plena y, con ello, ser independientes y capaces de tomar sus propias decisiones, la posibilidad de disfrutar y estar seguros, todo ello potenciando su crecimiento personal y su concepto de bienestar personal ${ }^{(13,14)}$.

Cuando se habla del espacio sociosanitario no hay que entenderlo como un tercer sector sino como un espacio compartido, un sistema complejo en el que se atiende a personas con necesidades sociales y sanitarias (12). Se trata de un ámbito en el que interactúan múltiples sectores, distintos niveles asistenciales, y que necesita de una coordinación entre ellos, para poder atender las diferentes necesidades de las personas mayores.

Esta realidad amplia de la visión de atención a los adultos mayores se ve reflejada en iniciativas conjuntas a nivel internacional como la Encuesta sobre salud, bienestar y envejecimiento (SABE) ${ }^{(15)}$, que investigó diferentes aspectos de salud de los adultos mayores de siete capitales de América Latina y el Caribe, de forma global desde un enfoque sociosanitario, con el objetivo de evaluar y comparar las realidades de las condiciones de salud y sus determinantes socioeconómicos de los países participantes, que sirvió de base para tomar acciones gubernamentales, como en el caso de Ecuador (16) que establece entre el 2007 y 2011 la política pública para las personas adultas mayores, que incluye una serie de programas con el fin de integrarlos al desarrollo del país a través de su participación activa. Así mismo, en comunidades como Durango en México ${ }^{(17)}$ se replicó la encuesta SABE que plantea una propuesta preliminar de un modelo estatal de atención al envejecimiento poblacional de Durango, y recalca la importancia de elaborar, desarrollar y consolidar estrategias gerontogeriátricas. A nivel intercontinental también se puede ver el efecto del enfoque sociosanitario en ejemplos como el programa EUROsociAL Salud ${ }^{(18)}$, iniciativa de la Comisión Europea que promueve la cohesión social en América Latina intercambiando experiencias europeas en diferentes ámbitos, entre ellos la salud; en ella participan comisiones de países como Francia, España, Chile, Argentina, Brasil, Italia; México y Costa Rica, buscando desarrollar políticas públicas de salud. 
Para el caso peruano, parte de esta estrategia es la llamada asistencia sociosanitaria como parte de una visión amplia, no solo del incremento de la cobertura prestacional de servicios de salud y sociales, estos últimos en fase reciente de expansión, desde la promoción social hasta la atención de largo plazo, con énfasis en el cuidado paliativo, permitiendo cubrir a las personas mayores en diferentes momentos dentro de su ciclo de vida, es decir, cuando tienen capacidad alta y estable, a personas mayores con deterioro de capacidad, y personas mayores con pérdida considerable de capacidad ${ }^{(19,20)}$.

A ello se han sumado estrategias de innovación social como parte de la contribución para el desarrollo del país a través del empoderamiento de nuestros adultos mayores (19) en sus derechos, su propio control de salud y autocuidado con participación de la familia, es por ello que creemos que este modelo será capaz de lograr cambiar estereotipos sociales negativos acerca de las diferentes formas de envejecer hacia una nueva mirada, que genere no solo una visión positiva del mismo sino interiorice herramientas que permitan tener envejecimientos positivos como parte de las nuevas generaciones de adultos mayores.

\section{ACCIONES SOCIOSANITARIAS DESARROLLADAS POR EL MINSA}

De acuerdo a los reportes del Ministerio de Salud (21) este organismo, a la fecha, viene implementando centros de atención integral para adultos mayores, conocidos como Tayta Wasi, en varias provincias del país, incluyendo Lima y Callao. Estos Tayta Wasi se encargan de brindar atención multidisciplinaria a la persona adulta mayor: médica, odontológica, nutricional, de terapia física, farmacia, y laboratorio, entre otras. Además, cuenta con clubes de adulto mayor en los que se realizan actividades preventivas promocionales y de educación en salud para sus integrantes.

Entre el 2013 y 2014 se implementaron 70 Tayta Wasi que beneficiaron alrededor de 77000 adultos mayores; la mayoría beneficiarios también del Programa Pensión 65. Al 2014, existían 2174 Clubes del Adulto Mayor en los 25 departamentos del país, lo que significó $18 \%$ más en relación al año anterior. En estos espacios participaron 67673 personas adultas mayores, $54 \%$ más que el año anterior ${ }^{(21)}$.

Respecto a las acciones sanitarias desarrolladas en el año 2014 (7):

- Con relación al aseguramiento por el SIS, se beneficiaron a 285214 personas adultas mayores (incremento en $22 \%$ respecto a 2013 ).
EI MINSA atendió a 1281654 personas adultas mayores, quienes recibieron, en promedio, 5,7 atenciones, con un 3,2\% más de atenciones en comparación al año 2013.

- En el marco del Plan de Intervención para la Rehabilitación Oral con Prótesis Removible en el Adulto Mayor 2012 - 2016 "Vuelve a sonreír", para el año 2014 se entregaron 4881 prótesis, a diferencia de los años 2012 y 2013 que registraron 7027 y 5743, respectivamente.

- Dentro del Plan de la Estrategia Sanitaria Nacional de salud ocular y Prevención de la ceguera evitable 2014-2020, se realizaron 14 971 cirugías en cataratas a personas adultas mayores en situación de pobreza y extrema pobreza.

- Se registraron 69103 atenciones en salud mental a personas adultas mayores, lo que representa un incremento del $2 \%$ más que el año anterior.

- En relación al Plan Esperanza -programa social del gobierno que atiende en forma integral los casos de cáncer- se realizaron 19 567 atenciones en el servicio de oncología en pacientes mayores de 60 años durante el periodo de 2011-2014. A través de campañas de salud MINSA-MIDIS, durante el 2014 se realizaron 2399 campañas de salud, con 147393 adultos mayores beneficiados.

\section{ACCIONES SOCIOSANITARIAS DESARROLLADAS EN ESSALUD}

EsSalud cuenta con un sistema de atención al asegurado bajo tres tipos de prestaciones: prestaciones sociales, prestaciones de salud y prestaciones económicas. Actualmente, como parte del proceso de envejecimiento del país, los asegurados también se han incrementado, los mayores de 60 años son 1095433 y los mayores de 80 años 256 000; lo que ha generado un alto impacto en la institución, por el incremento de las necesidades de cuidados que esta población requiere. Es por ello que se ha priorizado la condición de vulnerabilidad de dos poblaciones objetivo: persona adulta mayor y persona con discapacidad.

La diversidad en los servicios, que surge de las necesidades de salud de las personas mayores, como parte del proceso de envejecimiento, nos da la oportunidad de tener respuestas en políticas y servicios adecuados ${ }^{(22)}$. Por tal motivo, EsSalud ha migrado su estructura orgánica ${ }^{(23)}$ hacia la conceptualización del modelo del cuidado continuo e integrado a favor del bienestar de sus asegurados, entendido no solo como un modelo de atención a la enfermedad presente o no, sino también en la construcción de entornos favorables y seguros, centrados en las personas, dentro del buen 
gobierno corporativo y en el marco de la humanización. Dicha reorganización tiene articulados los cuidados continuados en las denominadas redes desconcentradas a nivel nacional.

\section{ACCIONES SOCIOSANITARIAS DE LOS SERVICIOS DE SALUD DE LAS FUERZAS ARMADAS Y POLICIALES}

Estas entidades ofrecen servicios de salud al personal militar o policial, incluyendo cónyuges e hijos, de acuerdo a la institución a la que pertenezca la persona. Existen cuatro sanidades que se encargan de brindar servicios asistenciales a este personal: Sanidad Militar, Sanidad de la Marina de Guerra, Sanidad de la Fuerza Aérea y Sanidad de las Fuerzas Policiales. Este grupo de centros fueron los primeros en crear servicios de atención especializada para adultos mayores, a la fecha cuentan con niveles de atención geriátrica adecuadamente establecidos y diferenciados ${ }^{(24)}$, en el que se da énfasis al enfoque multi- e interdisciplinario de la atención geriátrica. Una de estas instituciones emblemáticas, por ser el primero a nivel nacional en crearse, es el Hospital Geriátrico San José, fundado en 1999, la cual en el año 2015 tenía una población adulta mayor de 227845 personas. El hospital brinda los siguientes servicios ${ }^{(25)}$ :

Consulta externa especializada en cardiología, dermatología, otorrinolaringología, medicina física y rehabilitación, ginecología y traumatología; atiende $12 \mathrm{~h}$ al día.

Atención de emergencia las $24 \mathrm{~h}$. Cuenta con un área de observación para mujeres y varones y una unidad de intermedios.

Hospitalización de agudos. Brinda atención de pacientes con enfermedad aguda o reagudización de enfermedades crónicas, cuenta con 45 camas.

Hospital de día geriátrico (HODIGE). Servicio ambulatorio que completa la recuperación física, mental funcional y social del adulto mayor -independiente o con dependencia funcional leve- que previamente ha estado internado, con el fin de evitar reingresos y garantizar la continuidad de los cuidados. Realiza actividades como consejería a la familia, atenciones médicas, curaciones, talleres de manejo de emociones, de estimulación cognitiva, y de postura y equilibrio; funciona $6 \mathrm{~h}$ al día y cuenta con un médico geriatra, un técnico, una enfermera, una asistenta social, un psicólogo y un terapista físico.

Atención domiciliaria (ADOGE). Servicio de atención a adultos mayores con dependencia funcional, a los que se visita mensualmente, para asegurar la continuidad de cuidados en el domicilio, labora $12 \mathrm{~h}$ al día, atiende a 700 pacientes inscritos, cuenta con dos médicos geriatras permanentes y seis médicos generales capacitados en la atención a adultos mayores; seis enfermeras, seis terapistas físicos, una asistenta social, un psicólogo y un nutricionista, así como tres unidades móviles con sus respectivos conductores.

Además, cuenta con programas establecidos: Programa de Hipertensión Arterial, Programa de Diabetes Mellitus, Programa de Enfermedades Metabólicas, Programa de Consejería, Programa de Salud Mental y Violencia Familiar, Programa de Salud Bucal, Programa de Inmunizaciones, Programa de Fármacovigilancia. De igual manera, a nivel del resto de instituciones de las sanidades se toma en consideración las atenciones en estos servicios.

\section{CONCLUSIONES}

Los cambios sociodemográficos y epidemiológicos han conllevado a cambios en el enfoque de atención de salud de la población, sobre todo la población adulta mayor por sus características puntuales, debido a ello, a nivel político y gubernamental se vienen dando condiciones que han ampliado los servicios sociosanitarios con el fin de garantizar una mejor calidad de vida, esto, actualmente, permite tener a disposición mejores métodos de atención a adultos mayores en los diferentes niveles asistenciales.

\section{Fuentes de financiamiento: autofinanciado}

Conflictos de interés: los autores declaran no tener conflictos de interés.

Contribuciones de los autores: HCJ y PCV han participado en la concepción y diseño del artículo. RAP, JCD, HCJ y PCV contribuyeron en la redacción y aprobación final del artículo.

\section{REFERENCIAS BIBLIOGRÁFICAS}

1. Gálvez M, Olascoaga A. Demografía del envejecimiento: la transición demográfica. En: Principios de geriatría y gerontología. Lima: Centro Editorial UPCH; 2011. p. 25-33.

2. Organización Mundial de la Salud.
Informe Mundial sobre el Envejecimiento y la Salud. Ginebra: OMS; 2015.

3. Avellaneda A, Izquierdo M, TorrentFarnel J, Ramón JR. Enfermedades raras: enfermedades crónicas que requieren un nuevo enfoque socio sanitario. An Sist Sanit Navar. 2007;30(2):177-90.

4. Instituto Nacional de Estadística e Informática (INEI). Compendio estadístico 2015. Lima: INEI; 2015.

5. Congreso de la República. Ley de las 
personas adultas mayores. Ley 28803. Lima: Congreso de la República; 2006.

6. Ministerio de la Mujer y Poblaciones Vulnerables (MIMP). Plan Nacional para las personas adultas mayores 2013 2017. Lima: MIMP; 2011.

7. Ministerio de la Mujer y Poblaciones Vulnerables, Ministerio de Salud. III Informe anual de seguimiento al cumplimiento de la Ley de las Personas Adultas Mayores. Periodo 2014. Lima: MIMP/MINSA; 2014.

8. Ministerio de desarrollo e Inclusión social (MIDIS). Programa Nacional de Asistencia Solidaria-Pensión 65. Manual de Operaciones. Lima: MIDIS; 2012.

9. Ministerio de desarrollo e Inclusión social (MIDIS). Boletín electrónico de Pensión 65. Edición 21 de Julio 2014 [Internet]. Lima: MIDIS; 2014 [citado el 17 de enero de 2016]. Disponible en: http://www.pension65.gob. pe/2014/07/boletin-electronico-depension-65-edicion-21-de-julio-2014/

10. Ministerio de la Mujer y Poblaciones Vulnerables (MIMP). Programa nacional Vida Digna. Manual de Operaciones. Lima: MIMP; 2014.

11. Ministerio de la Mujer y Poblaciones Vulnerables (MIMP). Logros del Programa Nacional Vida Digna (periodo 2015) [Internet]. Lima: MIMP; 2015 [citado el 17 de enero de 2016]. Disponible en: http://www.mimp. gob.pe/vidadigna/index.php?option $=$ com_content $\&$ view $=$ article\&id $=142 \&$ Itemid $=573$

12. McLaughlin SJ, Jette AM, Connell CM. An examination of healthy aging across a conceptual continuum: prevalence estimates, demographic patterns, and validity. J Gerontol A Biol Sci Med Sci. 2012;67(7):783-9. doi: $10.1093 /$ gerona/glr234.
13. Bowling A, Dieppe P. What is successful ageing and who should define it? BMJ. 2005;331(7531):1548-51. doi: http:// dx.doi.org/10.1136/ bmj.331.7531.1548.

14. Ham C. The ten characteristics of the high-performing chronic care system. Health Econ Policy Law. 2010;5(Pt. 1):71-90. doi: 10.1017/ S1744133109990120.

15. Albala C, Lebrão ML, León Díaz EM, Ham-Chande R, Hennis AJ, Palloni $A$, et al. Encuesta Salud, Bienestar y Envejecimiento (SABE): metodología de la encuesta y perfil de la población estudiada. Rev Panam Salud Publica. 2005;17(5-6):307-22.

16. Ministerio de Inclusión económica y social (MIES). Agenda de Igualdad para adultos mayores 2012-2013. $2^{\mathrm{a}} \mathrm{ed}$. Quito: MIES; 2012.

17. Chavez-Mora O, Tinoco K, Martinez Y, Estrada S, Salvador J, Arroyo MC, Gonzales A. Propuesta preliminar de un modelo estatal de atención al envejecimiento poblacional de Durango. En: Encuesta Salud, bienestar y envejecimiento SABE. Durango. México, D.F.: Centro Nacional de Programas Preventivos y Control de Enfermedades; 2015.

18. Organización Iberoamericana de Seguridad Social. Extensión de la protección social en salud a poblaciones en condiciones especiales de vulnerabilidad (adulto mayor y dependencia). Documento Técnico. Madrid: Consorcio EUROsociAL Salud; 2008.

19. Low LF, Yap M, Brodaty H. A systematic review of different models of home and community care services for older persons. BMC Health Serv Res. 2011;11:93. doi: 10.1186/1472-696311-93.
20. Kornfeld-Matte R, editor. United Nations Office of the High Commisioner for Human Rights [Internet]. New York: United Nations; 2014 [citado el 17 de enero de 2016]. Disponible en: http://www.ohchr.org/ EN/NewsEvents/Pages/DisplayNews. aspx $?$ NewsID $=15148 \&$ LangID $=\mathrm{E}$

21. Ministerio de Salud del Perú. Tayta Wasi implementó el MINSA para prevenir el Alzheimer en el adulto mayor. Sala de Prensa [Internet]. Lima: MINSA; setiembre 2015 [citado el 17 de enero de 2016]. Disponible en: http://www. minsa.gob.pe $/$ ?op $=51 \&$ nota $=16916$.

22. EsSalud. Nueva Estructura Orgánica y Reglamento de Organización y Funciones del Seguro Social de Salud Resolución de Presidencia Ejecutiva $\mathrm{N}^{\circ}$ 656-PE-EsSalud-2014 [Internet]. Lima: EsSalud; 2014 [citado el 17 de enero de 2016]. Disponible en: http:// www.essalud.gob.pe/transparencia/ pdf/rof/resol_656_rof_dic_2014.pdf

23. Carstensen LL. The influence of a sense of time on human development. Science. 2006;312(5782):1913-5. doi: http:// dx.doi.org/10.1126/science.1127488.

24. Varela L, Chávez H, Herrera A, Méndez F, Gálvez M. Desarrollando respuestas integradas en sistemas de salud de una población en rápido envejecimiento. INTRA III. Lima; OPS/OMS; 2005.

25. Hospital Geriátrico PNP San José [Internet]. Lima: Hospital Geriátrico; 2015 [citado el 17 de enero de 2016]. Disponible en: http://hgsipnp.blogspot.es/

Correspondencia: Paola Casas Vásquez

Dirección: Av. Honorio Delgado 430, Urb. Ingeniería, San Martin de Porres. Lima, Perú. Teléfono:(511)987810148-(511)3190007 anexo 2246.

Correo electrónico: igero@oficinas-upch.pe, paola.casas.v@upch.pe

\section{Nuestros artículos se encuentran indizados en:}

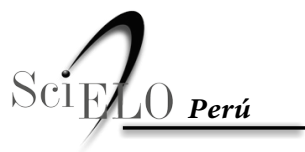

www.scielo.org 